\title{
P300: Waves Identification with and without Subtraction of Traces
}

\author{
Ana Carla Leite Romero ${ }^{1,2}$ Ana Cláudia Mirândola Barbosa Reis ${ }^{1} \quad$ Anna Caroline Silva de Oliveira ${ }^{2}$ \\ Humberto de Oliveira Simões ${ }^{3}$ Cinthia Amorim de Oliveira Junqueira ${ }^{4}$ Ana Cláudia Figueiredo Frizzo ${ }^{2}$
}

1 Department of Ophthalmology, Otorhinolaryngology and Head and Neck Surgery, Ribeirão Preto School of Medicine, Universidade de São Paulo, Ribeirão Preto, SP, Brazil

2 Department of Phonoaudiology, School of Philosophy and Sciences, Universidade Estadual Paulista Julio de Mesquita Filho, Marilia Campus, Marilia, SP, Brazil

3 Department of Neuroscience and Behavioral Sceinces, Universidade de São Paulo, Ribeirão Preto School of Medicine, Universidade de São Paulo, Ribeirão Preto, SP, Brazil

4 Department of Phonoaudiology, Universidade de Sao Paulo Campus de Ribeirao Preto, Ribeirao Preto, São Paulo, SP, Brazil
Address for correspondence Ana Cláudia Figueiredo Frizzo, MD, Fonoaudiologia, UNESP, Av. Hygino Muzzi Filho, 737, Marília, São Paulo 17.525-000, Brazil (e-mail: anafrizzo@marilia.unesp.br).

\author{
Abstract \\ Keywords \\ - electrophysiology \\ - evoked potentials \\ - auditory \\ - event-related \\ potentials \\ - P300 \\ - methods
}

Introduction The P300 test requires well-defined and unique criteria, in addition to training for the examiners, for a uniform analysis of studies and to avoid variations and errors in the interpretation of measurement results.

Objectives The objective of this study is to verify whether there are differences in P300 with and without subtraction of traces of standard and nonstandard stimuli.

Method We conducted this study in collaboration with two research electrophysiology laboratories. From Laboratory 1 , we selected 40 tests of subjects between 7-44 years, from Laboratory 2 , we selected 83 tests of subjects between 18-44 years. We first performed the identification with the nonstandard stimuli; then, we subtracted the nonstandard stimuli from the standard stimuli. The examiners identified the waves, performing a descriptive and comparative analysis of traces with and without subtraction.

Results After a comparative analysis of the traces with and without subtraction, there was no significant difference when compared with analysis of traces in both laboratories, within the conditions, of right ears ( $p=0.13$ and 0.28 for differences between latency and amplitude measurements) and left ears ( $p=0.15$ and 0.09 for differences between latency and amplitude measurements) from Laboratory 1 . As for Laboratory 2 , when investigating both ears, results did not identify significant differences ( $p=0.098$ and 0.28 for differences between latency and amplitude measurements). Conclusion There was no difference verified in traces with and without subtraction. We suggest the identification of this potential performed through nonstandard stimuli. received

September 2, 2016 accepted

December 21, 2016

published online

February 28, 2017
DOI https://doi.org/

10.1055/s-0037-1599096. ISSN 1809-9777.
Copyright $(2017$ by Thieme Revinter

Publicações Ltda, Rio de Janeiro, Brazil
License terms

(요 (1) $\Theta \circledast$ 


\section{Introduction}

P300 is an auditory-evoked potential denominated endogenous because it reflects the functional use individuals make of the auditory stimulus, being highly dependent on cognitive abilities, including attention and auditory discrimination. It is an objective procedure which depends on the examiner's experience in detecting the peaks of the waves. A methodology for the identification of traces that facilitates the analysis of presence of response and interpretation of results is relevant in this case. ${ }^{1}$

The P300 potential provides a window to observe the neurophysiological substrate of processes that occur in the cerebral cortex related to cognition, memory, and auditory attention. ${ }^{2}$ The feature is the most often reported in research focusing on hearing. However, latency and amplitude measures are variable and must be adjusted to age and to the studied population. $^{3}$

For the P300 analysis, examiners should take into account possible interference that contribute to its variability, such as the parameters used in the test (intensity, frequency and type of acoustic stimulus evoking, filter, type of task, interstimuli interval, among others), the identification conditions (time of day), the subject (age/maturity, sex, cognitive ability, body temperature), and the examiner's skills for verifying such a potential. ${ }^{4}$

According to Schochat, ${ }^{5}$ P300 is identified as a wave of positive polarity with latency of $\sim 300$ milliseconds poststimulus, obtained after subtracting the trace corresponding to nonstandard stimuli from the trace corresponding to standard stimuli.

Recent research with diverse populations have performed the identification of P300 in nonstandard traces, without performing the subtraction from the standard trace, and have found reliable results..$^{6-8}$

Although P300 is considered an objective test, a single, well-defined criteria, and training of its examiners is essential for a more uniform analysis of studies, and a verification whether or not subtraction of the traces is necessary to benefit from these potentials.

In this context, our study aims to determine whether there is difference in P300 with and without subtraction.

\section{Material and Method}

This study received approval from the Research Ethics Committees of both institutions involved in this research under the Research protocols Number 842/2010 and 12790/2011, respectively.

This is a documental, experimental, and descriptive study. It was a partnership between two Electrophysiology Research Laboratories in Hearing.

The examiners of both services were experienced in Auditory Electrophysiology, specifically in Long Latency Auditory Evoked Potentials.

From Laboratory 1, we selected and analyzed 40 cognitive auditory evoked potential tests of subjects ( 24 women and 16 men) with normal development, previously performed in participating volunteers from previous research in the same field. The subjects were of both sexes, aged between 7 and 44 years, without cognitive impairment, according to history available in medical records, and audiometric thresholds within the normal range $\left(20\right.$ a $\left.30 \mathrm{dBHL}^{9}\right)$.

From Laboratory 2, we selected and analyzed 83 cognitive auditory evoked potential tests of subjects ( 44 women and 30 men) with normal development and audiometric thresholds within the normal range $\left(20 \mathrm{dBHL}^{9}\right)$, previously performed in participating volunteers from previous research in the same field. The study subjects were men and women aged 18-44 years.

In Laboratory 1, for P300 assessment, we used Biologic Navigator Pro and captured the responses with active electrode in the central region $\mathrm{Cz}$, referring to the electrodes positioned in the right lobe (A2) and left (A1) and the ground electrode at the forehead (Fpz). In Laboratory 2, we used 3A insert phones, the acoustic stimulus was tone-burst at 70 $\mathrm{dBnHL}$, presented randomly by the computer, at a proportion of $20 \%$ nonstandard stimuli of a total of 200 stimuli, identified in a 500 milliseconds window, $100.000 \mathrm{x}$ amplification, alternating polarity, with bandpass filtering of $0.5-30 \mathrm{~Hz}$, monaural stimulation, and stimulation rate of 1.1 /second.

For frequency discrimination, we used a standard stimulus at a $750 \mathrm{~Hz}$ frequency and nonstandard stimulus at $1000 \mathrm{~Hz}$ frequency with $20 \mathrm{~ms}$ - rise/fall and $60 \mathrm{~ms}$ - plateau, both with duration of $100 \mathrm{~ms}$. Subjects were instructed to pay attention and identify random nonstandard stimuli within a series of standard stimuli and name them out loud: fine to $1000 \mathrm{~Hz}$.

In Laboratory 2, for the P300 evaluation, we used Bio-Logic equipment, version 5.70, model 317 (Bio-Logic Science Instruments S.A.S, Seyssinet-Pariset, France). We captured responses with active electrodes positioned at $\mathrm{Cz}$ and $\mathrm{Fz}$ and connected to the input of a preamp channels 1 and 2 , respectively. We placed the reference electrodes on the earlobe (A1 and A2), interconnected and connected to input 2 of channel 1, and interconnected to channel 2 by the preamplifier jumper. We placed the ground electrode at the forehead (Fpz). We used $3 \mathrm{~A}$ insert phones, the acoustic stimulus was presented through toneburst at $75 \mathrm{dBnHL}$, binaurally, presented randomly at a proportion of $20 \%$ stimuli of a total of 240 stimuli, identified in a $500 \mathrm{~ms}$ window, amplification $100.000 \mathrm{x}$, alternating polarity, with band filtering of $1-30 \mathrm{~Hz}$ and 1.1 stimuli per second stimulation rate.

For frequency discrimination, we used a standard stimulus at $1000 \mathrm{~Hz}$ frequency and nonstandard stimuli at $2000 \mathrm{~Hz}$ frequency with $20 \mathrm{~ms}$ rise/fall and $60 \mathrm{~ms}$ plateau, both with duration of $100 \mathrm{~ms}$. Subjects were instructed to pay attention and identify the nonstandard stimuli (random) within a series of standard stimuli, counting mentally.

The tests took place with two successive passages to allow good definition and replication. Impedance was maintained at $3 \mathrm{~kW}$ or less.

The tests lasted $\sim 50$ minutes. As a protocol to ensure quality examination, we suggested to those with myogenic interference that they change position. We also ensured quality by controlling the answers for a maximum of $5 \%$ deviation between stimuli data and patient responses and, 
when necessary, repeating the test. Also, we asked the subjects to keep their eyes closed to eliminate eye artifacts.

For the identification of P300 waves, we instructed all examiners to use the criterion proposed by Junqueira and Colafêmina ${ }^{4}$ adapted as follows: identification of N1-P2-N2 complex - the first three waves that appear in sequence - and present the polarities: negative - positive - negative, respectively, occurring in the replication of traces, standard and nonstandard between 60 and 300ms; P3 identification, the highest positive wave, right after N1-P2-N2 complex, occurring in trace replication to nonstandard stimulus, between 240 and $700 \mathrm{~ms}$. We identified latencies at the highest peak, that is, the maximum wave amplitude point. We identified amplitudes at the wave peak to the base line and interamplitude in the case of N2-P3 inter-amplitude; when there was duplication of P3 wave in P3a and P3b, we always performed the identification in the second wave, P3b. ${ }^{10}$ In addition, the identification of waves always occurred in the identification of the nonstandard stimuli.

We first analyzed and identified the tests for nonstandard stimulus, then performed a second identification by subtracting the nonstandard stimulus from the standard stimulus. Examiners were instructed according to pre-established criteria for identifying the cognitive potential waves.

In this study, we chose to focus on the analysis of P300 with and without subtraction, since this has been the most used analysis in research involving auditory electrophysiological assessments.

As a study that analyzes the intersubject response, we did not consider small variations of the protocols in the services as variables that could affect the results and objectives of this study.

We performed a descriptive analysis of the test results, and then conducted a comparative analysis using Student $t$-test among the variables of P300 latency and amplitude, comparing these variables identified in the traces with subtraction and without subtraction. We only analyzed the active electrode arrangement positioned in $\mathrm{C} z$, a position that ensures records with better morphology and greater amplitude. ${ }^{11}$

We describe results as p-value, and the significance level was always $5 \%$ or 0.05 ( $\mathrm{p} \leq 0.05$ ). The statistics software used was Bioestat (Instituto de Desenvolvimento Sustentável Mamirauá, Tefé, Brazil).

\section{Results}

-Table 1 shows the mean value, standard deviation, and p-value of P300 latency and amplitude, respectively, from Laboratories 1 and 2 after statistical analysis through $t$-test. We found no significant differences between the measures with and without subtraction.

-Figs. 1 and 2 present a representative box plot comparing the measures with and without subtraction from both laboratories.

\section{Discussion}

LLAEP is one of the most promising measures in objective auditory assessment as it comprehends from simple listening skills to the most complex ones. According to Magliaro, ${ }^{12}$ auditory-evoked potentials have been useful tools for functional diagnostics, allowing one to observe, through an increase in latency or decrease in amplitudes, objective evidence of clinical and sub-clinical problems.

This potential's analysis, although an objective procedure, is highly subjective, depending on good clinical experience to visually detect the waves. ${ }^{1}$ Inaddition, standardizationsfor their identification are required to avoid variations of traces and difficultiesintheinterpretationofthestudy.Oneoftheprocedures for interpreting the results is whether there is variation when traces are analyzed with subtraction or no subtraction of the nonstandard stimulus wave from the standard stimulus wave.

Table 1 Descriptive statistics and p-values: $t$ test of P300 latency and amplitude of measures with and without subtraction

\begin{tabular}{|c|c|c|c|c|c|c|c|c|c|c|}
\hline Group & Ear & Analysis & Variable & Mean & SD & Min & $\begin{array}{l}\text { 1st Quartile } \\
(25 \%)\end{array}$ & $\begin{array}{l}\text { 3rd Quartile } \\
(75 \%)\end{array}$ & Max & $\begin{array}{l}p \text { Value } \\
\text { t Test }\end{array}$ \\
\hline \multirow[t]{8}{*}{ Laboratory 1} & \multirow[t]{4}{*}{$\mathrm{R}$} & \multirow[t]{2}{*}{ with sub } & lat_P300 & 335.82 & 32.35 & 275 & 308 & 352.50 & 246 & \multirow[t]{2}{*}{0.136} \\
\hline & & & lat_P300 & 339.78 & 35.40 & 246 & 319 & 356 & 415 & \\
\hline & & \multirow[t]{2}{*}{ without sub } & amp_P300 & 6.25 & 2.77 & 2 & 4 & 8 & 2 & \multirow[t]{2}{*}{0.286} \\
\hline & & & amp_P300 & 6.59 & 2.69 & 13 & 4 & 8 & 14 & \\
\hline & \multirow[t]{4}{*}{$\mathrm{L}$} & \multirow[t]{2}{*}{ with sub } & lat_P300 & 327.17 & 35.29 & 274 & 301.5 & 346.50 & 411 & \multirow[t]{2}{*}{0.158} \\
\hline & & & amp_P300 & 330.44 & 41.92 & 246 & 304 & 355.75 & 416 & \\
\hline & & \multirow[t]{2}{*}{ without sub } & lat_P300 & 5.69 & 3.01 & - & 4 & 7 & 12 & \multirow[t]{2}{*}{0.095} \\
\hline & & & amp_P300 & 5.81 & 3.12 & - & 4 & 8 & 15 & \\
\hline \multirow[t]{4}{*}{ Laboratory 2} & \multirow[t]{4}{*}{$\mathrm{BIN}$} & \multirow[t]{2}{*}{ with sub } & lat_P300 & 289.90 & 34.02 & 225 & 264 & 315 & 371 & \multirow[t]{2}{*}{0.095} \\
\hline & & & amp_P300 & 296.89 & 34.33 & 237 & 271 & 325 & 409 & \\
\hline & & \multirow[t]{2}{*}{ without sub } & lat_P300 & 7.15 & 4.10 & - & 4 & 9.50 & 21 & \multirow[t]{2}{*}{0.280} \\
\hline & & & amp_P300 & 7.46 & 3.82 & 1 & 5 & 10 & 22 & \\
\hline
\end{tabular}

Abbreviations: R, right; L, left; BIN, bineural; amp, amplitude ( $\mu$ V); SD, standard deviation; lat, latency (ms); sub, subtraction. 


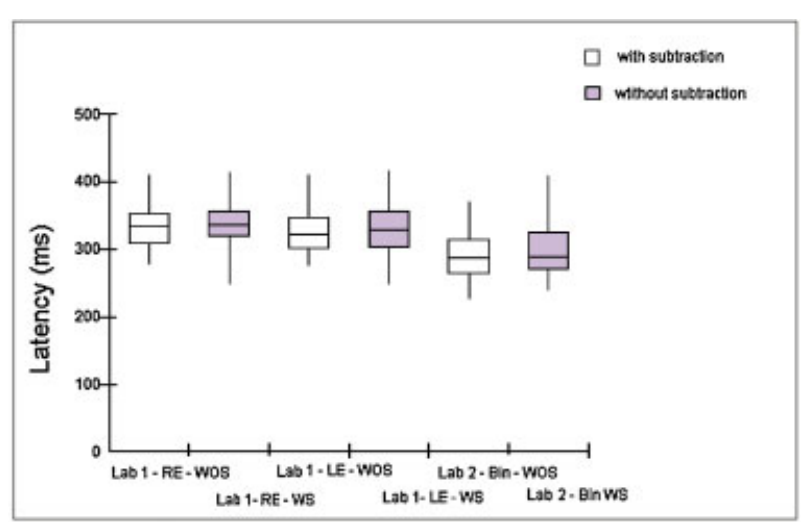

Fig. 1 Box plot of latency measurements: comparison with subtraction and without subtraction. Abbreviations: Bin, Binaural; LE, Left ear; RE, Right ear; WOS, without subtraction; WS, with subtraction.

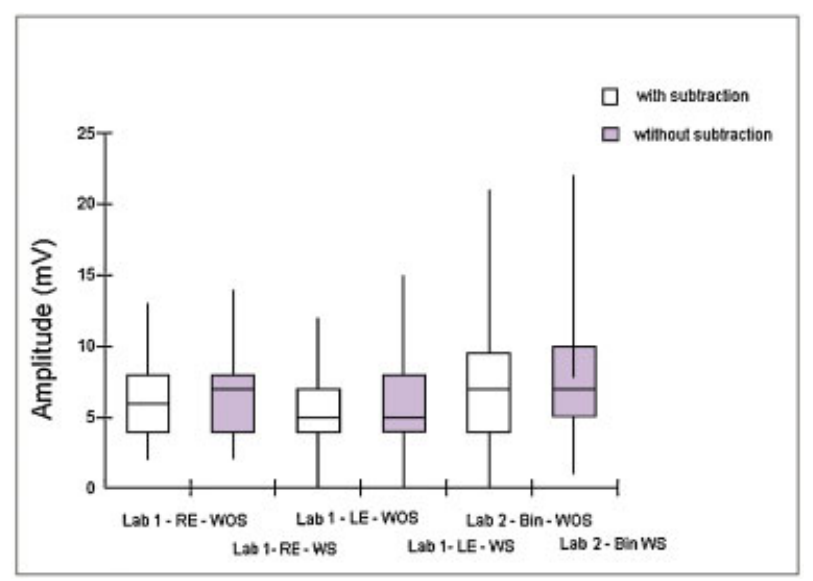

Fig. 2 Box plot of amplitude measurements: comparison with subtraction and without subtraction. Abbreviations: Bin, Binaural; LE, Left ear; RE, Right ear; WOS, without subtraction; WS, with subtraction.

In this study, after a comparative analysis of the traces with and without subtraction, we found that none of the variables showed statistically significant results, suggesting nodifference in the identification of traces with and without subtraction.

Colin ${ }^{13}$ performed the identification of the P300 wave from the subtraction of the trace corresponding to nonstandard stimuli in comparison to the trace corresponding to standard stimuli, and identified the wave as having positive polarity $\sim 300$ milliseconds after stimulus.

Näätänen et $\mathrm{al}^{14}$ state that this subtraction is needed to obtain the Mismatch Negativity (MMN), which is an electrical brain response triggered by a discernible change in any repetitive aspect of auditory stimulation, that is, between the neural representation developed by the standard stimulus in memory and sensory input of the nonstandard stimulus. The system gets used to the standard stimulus, therefore fewer neurons trigger synapses in response to this stimulus in comparison with the nonstandard stimulus, which, because heard less often, generates more synapses, which in turn generate a wave of greater amplitude, the MMN.

Matas, ${ }^{6}$ Soares, ${ }^{7}$ and Rabelo ${ }^{8}$ performed P300 identification in nonstandard stimulus trace without performing the subtraction in relation to the standard stimulus, and found reliable results concerning P300 with the identification on the traces related to nonstandard stimuli.

In this context, we suggest that the identification of this potential be performed for the nonstandard stimulus trace, given that we observed no statistically significant differences when subtraction was performed from the standard stimuli. In addition, further studies are warranted to confirm these findings.

\section{Conclusion}

In this study, after a comparative analysis of the traces with and without subtraction, we found that none of the variables showed statistically significant results, suggesting no difference in identifications of traces with and without subtraction. Thus, we suggest that identification of this potential be conducted for nonstandard stimulus traces.

\section{References}

1 Duarte JL. Potencial evocado auditivo de longa latência-P300 em indivíduos normais: valor do registro simultâneo em Fz e Cz. Rev Bras Otorrinolaringol 2009;75(02):231-236

2 Sousa LCA, Piza MRT, Alvarenga KF, Cóser PL. Eletrofisiologia da audição e emissões otoacústicas: princípios e aplicações clínicas. São Paulo: Tecmedd; 2008a. Chap. 2. Conceitos básicos e fundamentos neurofisiológicos;7-8

3 Hoshii LA. Potencial evocado auditivo de longa latência em um grupo de crianças deficientes auditivas usuárias de aparelho de amplificação sonora indivídual: estudo de caso [dissertation]. Campinas: PUC-SP; 2010:145

4 Junqueira CAO, Colafêmina JF. Investigation of inter- and intraexaminer stability to P300 auditory identification: analysis of errors. Rev Bras Otorrinolaringol 2002;68(04):468-478

5 Schochat E. Respostas de Longa Latência. Em: Carvallo, RMM, organizadora. Fonoaudiologia: Informação para Formação - Procedimentos em Audiologia. Rio de Janeiro: Editora Guanabara Koogan; 2003:71-7

6 Matas CG, Hataiama NM, Gonçalves IC. Estabilidade dos potenciais evocados auditivos em indivíduos adultos com audição normal. Rev Soc Bras Fonoaudiol 2011;16(01):37-41

7 Soares AJC, Sanches SGG II, Neves-Lobo IF, Carvallo RMM, Matas CG, Cárnio MS. Potenciais evocados auditivos de longa latência e processamento auditivo central em crianças com alterações de leitura e escrita: dados preliminares. Arq Int Otorrinolaringol 2011;15(04):486-491

8 Rabelo CM, Neves-Lobo IF, Rocha-Muniz CN, Ubiali T, Schochat E. Cortical inhibition effect in musicians and non-musicians using P300 with and without contralateral stimulation. Rev Bras Otorrinolaringol (Engl Ed) 2015;81(01):63-70

9 Llloyd LL, Kaplan H. Audiometric interpretation: a manual of basic audiometry. University Park Press: Baltimore; 1978;94:16-7

10 Polich J. Bifurcated P300 peaks: P3a and P3b revisited? J Clin Neurophysiol 1988;5(03):287-294

11 Polich J. Normal variation of P300 from auditory stimuli. Electroencephalogr Clin Neurophysiol 1986;65(03):236-240

12 Magliaro FCL, Scheuer CI, Assumpção Júnior FB, Matas CG. Study of auditory evoked potentials in autism. Pro Fono 2010;22(01):31-36

13 Colin C. Avaliação comportamental e eletrofisiológica das funções auditivas no processo do envelhecimento [dissertation]. São Paulo: Faculdade de Medicina da Universidade de São Paulo; 2013:129

14 Näätänen R, Paavilainen P, Rinne T, Alho K. The mismatch negativity (MMN) in basic research of central auditory processing: a review. Clin Neurophysiol 2007;118(12):2544-2590 